\title{
TROMBÓLISE EM TROMBOEMBOLISMO PULMONAR DE RISCO INTERMEDIÁRIO
}

DOI: $10.5380 /$ rmu.v1i3.40812

Valéria Cristina Scavasine ${ }^{1}$, Fernando Michiellin¹, Anderson Roberto Dallazen ${ }^{1}$, Gustavo Lenci Marques ${ }^{1}$, Maurício Carvalho ${ }^{1}$ RESUMO

Tromboembolismo pulmonar (TEP) submaciço caracteriza-se por disfunção ventricular direita ou elevação de marcadores de necrose miocárdica em pacientes normotensos. A melhor terapêutica para esses casos permanece controversa. Há evidências recentes na literatura apontando para o benefício da terapia com fibrinolítico em pacientes com baixo risco de sangramento. Apresentamos o caso de uma jovem de 17 anos com TEP submaciço, submetida a trombólise com alteplase, com excelente evolução clínica.

INTRODUÇÃO

O TEP é uma emergência cardiovascular relativamente comum, com formas diversas de apresentação clínica. Foram relatadas incidências de 23 a 69 casos por 100.000 habitantes por ano em estudos norte-americanos. ${ }^{8,10}$ É frequentemente fatal, porém o diagnóstico precoce e o tratamento específico podem mudar dramaticamente o desfecho clínico. ${ }^{9}$

O TEP pode ser classificado como maciço, submaciço ou de baixo risco. A presença de choque cardiogênico define o evento maciço e requer cuidados intensivos imediatos e trombólise. ${ }^{5} \mathrm{O}$ maior benefício do agente fibrinolítico ocorre nas primeiras 48 horas após instalação do quadro. Porém, há relatos de que ainda pode ser eficaz mesmo após 14 dias de sintomas. ${ }^{2}$

O TEP submaciço, caracterizado por insuficiência ventricular direita ou elevação de troponina, figura como uma área nebulosa na literatura médica, na medida em que a abordagem ideal desses pacientes permanece controversa. ${ }^{6}$

O TEP pode ser a primeira manifestação de uma trombofilia hereditária ou adquirida; porém, antes de solicitar diversos exames laboratoriais de alto custo, o clínico deve estar atento para as indicações, momento ideal para coleta e interpretação desses resultados.

\section{RELATO DO CASO}

Paciente do sexo feminino, 17 anos, previamente hígida, apresenta-se à unidade de emergência com queixa de dor retroesternal, subcostal direita e interescapular, de caráter ventilatório dependente, associada à dispneia súbita durante o banho, taquipneia, tosse não produtiva e taquicardia. Em uso de anticoncepcional hormonal oral (etinilestradiol $35 \mathrm{mcg}$ e ciproterona $2 \mathrm{mg}$, Selene ${ }^{\circledR}$ ) há dois meses. Ao exame de admissão apresentava-se em bom estado geral, não cianótica, afebril, pressão arterial (PA) de 132/90 $\mathrm{mmHg}$, frequência cardíaca (FC) de 137 batimentos por minuto, frequência respiratória (FR) de 22 movimentos respiratórios por minuto, saturação periférica de oxihemoglobina (SpO2) de 89\% em ar ambiente, ausência de ingurgitamento de jugulares, sem alterações aos exames cardiológico e pulmonar, ausência de visceromegalias e sem edema de extremidades.

Diante da suspeita de tromboembolismo pulmonar (TEP), foi internada na Unidade Coronariana do HC. Realizou eletrocardiograma, que demonstrou taquicardia sinusal; ecocardiograma, que revelou disfunção ventricular direita (dimensão diastólica de 32 $\mathrm{mm}$ e hipocinesia médio-basal, sinal de McConnell) e

\author{
1-Universidade Federal do Paraná \\ Contato do Autor / Mail to: \\ Valéria Cristina Scavasine - valeriascavasine@hotmail.com \\ Rua General Carneiro, 181 - Alto da Glória Curitiba - PR, 80060-900, 10 Andar, Departamento de Clínica Médica
}


elevação da pressão sistólica da artéria pulmonar (PSAP, $79 \mathrm{mmHg}$ ). A angiotomografia pulmonar evidenciou trombo volumoso em artéria pulmonar direita e nas artérias segmentares esquerdas (Figura 1). Durante a investigação laboratorial (Tabela 1) detectou-se elevação de troponina I de alta sensibilidade (232 $\mathrm{pg} / \mathrm{dL})$.

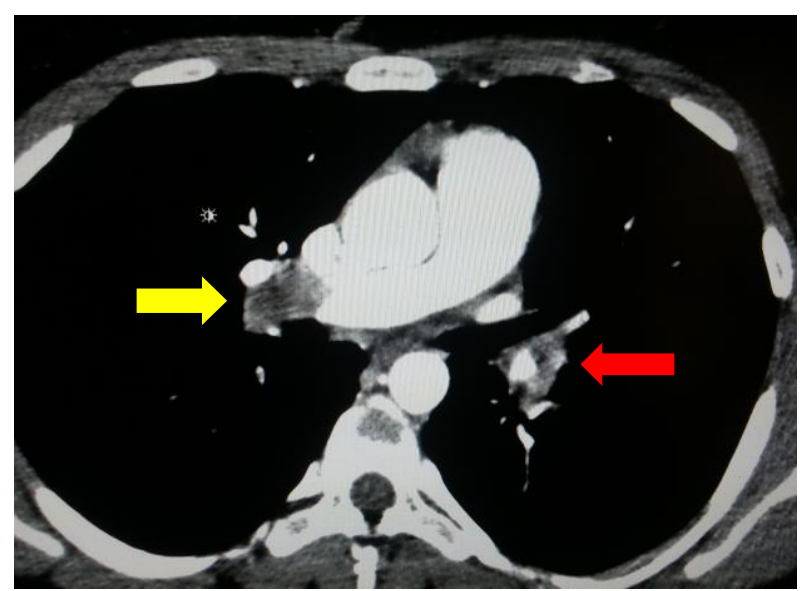

Figura 1. Tomografia contrastada de tórax mostrando trombos volumosos em artéria pulmonar direita (seta amarela) e segmentares esquerdas (seta vermelha).

Tabela 1. Investigação laboratorial

\begin{tabular}{|c|c|c|}
\hline & Paciente & $\begin{array}{l}\text { Valor de } \\
\text { referência }\end{array}$ \\
\hline $\mathrm{pH}$ & 7,5 & $7.35-7.48$ \\
\hline $\mathrm{PaO}_{2}$ & $64 \mathrm{mmHg}$ & $80-100 \mathrm{mmHg}$ \\
\hline Sat $\mathrm{O}_{2}$ & $94 \%$ & $94-100 \%$ \\
\hline $\mathrm{PaCO}_{2}$ & $24 \mathrm{mmHg}$ & $35-45 \mathrm{mmHg}$ \\
\hline $\mathrm{HCO}_{3}^{-}$ & $22,6 \mathrm{mEq} / \mathrm{L}$ & $22-25 \mathrm{mEq} / \mathrm{L}$ \\
\hline $\mathrm{Hb}$ & $15 \mathrm{~g} / \mathrm{dL}$ & $\begin{array}{c}12.0-15.5 \\
\mathrm{~g} / \mathrm{dL}\end{array}$ \\
\hline VG & $43,5 \%$ & $36.0-45.0 \%$ \\
\hline Leucócitos & $\begin{array}{l}15950 \\
/ \mathrm{mm}^{3}\end{array}$ & \\
\hline Bastões & $2 \%$ & \\
\hline Linfócitos & $1754 / \mathrm{mm}^{3}$ & \\
\hline Plaquetas & $\begin{array}{c}167 \\
000 / \mathrm{mm}^{3}\end{array}$ & $\begin{array}{c}140-400000 \\
\mathrm{~mm}^{3}\end{array}$ \\
\hline RNI & 1,00 & $0.8-1.2$ \\
\hline Glicemia & $96 \mathrm{mg} / \mathrm{dL}$ & até 99 mg/dL \\
\hline $\mathrm{Na}^{+}$ & $139 \mathrm{mEq} / \mathrm{L}$ & $\begin{array}{c}139-145 \\
\mathrm{mEq} / \mathrm{L}\end{array}$ \\
\hline
\end{tabular}

\begin{tabular}{|c|c|c|}
\hline & & Continuação \\
\hline $\mathrm{K}^{+}$ & $4,3 \mathrm{mEq} / \mathrm{L}$ & $\begin{array}{c}3.5 \text { a } 5.1 \\
\mathrm{mEq} / \mathrm{L}\end{array}$ \\
\hline Creatinina & $0,8 \mathrm{mg} / \mathrm{dL}$ & $\begin{array}{c}0.57-1.11 \\
\mathrm{mg} / \mathrm{dL}\end{array}$ \\
\hline Ureia & $19 \mathrm{mg} / \mathrm{dL}$ & 21 a $43 \mathrm{mg} / \mathrm{dL}$ \\
\hline Bilirrubinas totais & $0,41 \mathrm{mg} / \mathrm{dL}$ & $\begin{array}{c}0.1 \text { a } 1.2 \\
\mathrm{mg} / \mathrm{dL}\end{array}$ \\
\hline Bilirrubina direta & $0,24 \mathrm{mg} / \mathrm{dL}$ & $<0.2 \mathrm{mg} / \mathrm{dL}$ \\
\hline Bilirrubina indireta & $0,17 \mathrm{mg} / \mathrm{dL}$ & $<0.8 \mathrm{mg} / \mathrm{dL}$ \\
\hline ALT & $23 \mathrm{UI} / \mathrm{L}$ & $5-34 \mathrm{UI} / \mathrm{L}$ \\
\hline AST & $24 \mathrm{UI} / \mathrm{L}$ & $0-55$ UI/L \\
\hline $\begin{array}{l}\text { Troponina I de alta } \\
\text { sensibilidade }\end{array}$ & $232 \mathrm{pg} / \mathrm{dL}$ & $<15.6 \mathrm{pg} / \mathrm{dL}$ \\
\hline Albumina & $4,1 \mathrm{~g} / \mathrm{dL}$ & $2.9-5.2 \mathrm{~g} / \mathrm{dL}$ \\
\hline Lactato & $1,3 \mathrm{mg} / \mathrm{dL}$ & $0.5-2.0 \mathrm{mg} / \mathrm{dL}$ \\
\hline TSH & $\begin{array}{c}3,36 \\
\mathrm{uUI} / \mathrm{mL}\end{array}$ & $\begin{array}{c}0.35-4.94 \\
\mathrm{mg} / \mathrm{dL}\end{array}$ \\
\hline T4 livre & $1,24 \mathrm{pmol} / \mathrm{L}$ & $\begin{array}{c}1.7-1.48 \\
\mathrm{mg} / \mathrm{dL}\end{array}$ \\
\hline$\beta-H C G$ & $\begin{array}{c}<1,2 \\
\mathrm{mUl} / \mathrm{mL}\end{array}$ & $\begin{array}{c}\text { Gravidez }>25 \\
\mathrm{mUl} / \mathrm{mL}\end{array}$ \\
\hline
\end{tabular}

Confirmado o diagnóstico, foi heparinizada e optou-se pela realização de trombólise com o uso de Alteplase. Não foram registradas intercorrências durante o procedimento. A paciente foi então transferida para a enfermaria para anticoagulação com warfarina e investigação de trombofilia. Foram solicitados: proteína C ( $60 \%$ de atividade, referência 70 a $140 \%)$, antitrombina III (112\% de atividade, referência 75 a 125\%), anticorpo anticardiolipina (não reagente), FAN (1:160, padrão pontilhado fino), mutação G20210 A da protrombina (PCR em tempo real, ausência de mutação), anti-beta2 glicoproteína IgG e IgM (não reagentes), fator $\mathrm{V}$ de Leiden (PCR em tempo real, ausência de mutação R506Q) e homocisteína (7 Hmol/L, referência 4 a 10).

A paciente evoluiu bem, tornando-se assintomática. O ecocardiograma de controle revelou apenas discreta elevação da PSAP (30 mmHg) e normalização da função ventricular direita; eletrocardiograma sem alterações. Recebeu alta com orientações quanto à substituição do uso de contraceptivo hormonal oral pela adoção de método de barreira e prescrição de rivaroxaban $20 \mathrm{mg}$ ao dia. 


\section{DISCUSSÃO}

De acordo com a American Heart Association, o TEP é classificado em escala crescente de gravidade como de baixo risco, submaciço ou maciço ${ }^{4}$. O evento maciço é definido por choque, com pressão arterial sistólica abaixo de $90 \mathrm{mmHg}$ por pelo menos 15 minutos, ou com necessidade de inotrópicos. O TEP submaciço se caracteriza pela disfunção nova de ventrículo direito ou pela elevação de marcadores de necrose miocárdica. As tromboses de baixo risco, por sua vez, são aquelas não possuem nenhum dos comemorativos citados anteriormente.

A terapêutica muda substancialmente conforme a gravidade do TEP. Está bem estabelecida na literatura a trombólise como terapia de eleição para o TEP maciço, podendo ser realizada com Alteplase $10 \mathrm{mg}$ em bolus e $90 \mathrm{mg}$ em 2 horas, máximo de 1.5 $\mathrm{mg} / \mathrm{kg}$. Nos casos de baixo risco, a anticoagulação plena parece ser suficiente. No entanto, a conduta ideal no TEP submaciço permanece controversa.

Visando a preencher essa lacuna, um estudo coreano (Choi, 2009) ${ }^{1}$ criou o escore PESI (tabela 2) para estratificar os pacientes com TEP submaciço que poderiam se beneficiar de abordagens mais agressivas. De acordo com esse escore, pacientes com pontuação > 105 apresentariam maior risco de evolução para choque cardiogênico e, portanto, indicação mais precisa de fibrinolítico. Nossa paciente pontou 57, sendo considerada de muito baixo risco para instabilidade hemodinâmica.

Tabela 2. Escore PESI

\begin{tabular}{|l|c|}
\hline Preditor & Pontos \\
\hline Idade & $\begin{array}{c}\text { Cada ano, } \\
\text { um ponto }\end{array}$ \\
\hline Homem & +10 \\
\hline Câncer & +30 \\
\hline Pneumopatia crônica & +10 \\
\hline Insuficiência Cardíaca & +10 \\
\hline Pulso $>110$ bpm & +20 \\
\hline FR $>30$ ipm & +20 \\
\hline Temp < 36oC & +20 \\
\hline Rebaixamento de Nível de consciência & +60 \\
\hline Saturação $<90 \%$ & +20 \\
\hline
\end{tabular}

Tabela 2. Continuação

\begin{tabular}{l|l}
\hline$<65$ & Muito baixo risco \\
\hline $66-85$ & Baixo risco \\
\hline $86-105$ & Risco intermediário \\
\hline $106-125$ & Alto risco \\
\hline$>125$ & Muito alto risco \\
\hline
\end{tabular}

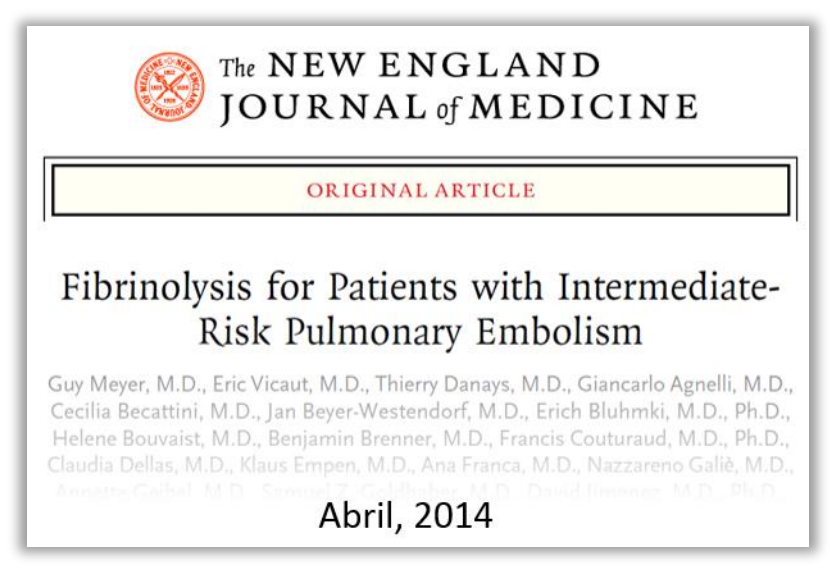

Figura 2 - New England Journal of Medicine

Em Abril de 2014, Meyer ${ }^{4}$ publicaram um ensaio clínico no New England Journal of Medicine (Figura 2), comparando os resultados obtidos com fibrinólise versus placebo no tromboembolismo pulmonar submaciço. Trata-se de um ensaio clínico duplo-cego, randomizado, conduzido no Hospital da Assistência Pública de Paris, que separou pacientes normotensos com TEP em dois grupos: um que recebeu tenecteplase mais heparina, e outro, com placebo mais heparina. Os desfechos analisados foram mortalidade em 90 dias, sangramento maior e choque cardiogênico.

Foram incluídos os pacientes com mais de 18 anos, com TEP confirmado por tomografia de tórax ou angiotomografia, além de disfunção de VD ao ecocardiograma ou evidências de necrose miocárdica. Foram excluídos os quadros de hipotensão ou hipertensão descontrolada, pacientes com alto risco de sangramento e pós-procedimentos, como filtro de veia cava, trombólise ou trombectomia mecânica há menos de 4 dias.

Do total de 1006 pacientes randomizados, 505 foram alocados para o grupo tenecteplase e 500 receberam o tratamento padrão (Figura 3). A intervenção foi realizada com tenecteplase, $10 \mathrm{mg} \mathrm{EV}$ em dose única, seguida por dose de manutenção com heparina não-fracionada. Dentre os pacientes que receberam fibrinolítico, 13 foram a óbito em 7 dias, representando $2.6 \%$ do total desse grupo; enquanto isso, no grupo placebo, obteve-se mortalidade de 5.6\%, totalizando 28 pacientes. A diferença foi significativamente estatística, com OR $=44$ e $p=0,02$.

Sangramento extracraniano ocorreu mais frequentemente no grupo tenecteplase, com $p<0,0001$; tais casos foram mais frequentes em pacientes idosas (acima de 75 anos) do sexo feminino. Este estudo, portanto, permitiu concluir que a trombólise com tenecteplase efetivamente reduz o risco de descompensação hemodinâmica ou morte nos pacientes com TEP submaciço; obviamente, deve ser usada com cautela nos grupos com maior risco de sangramento. 


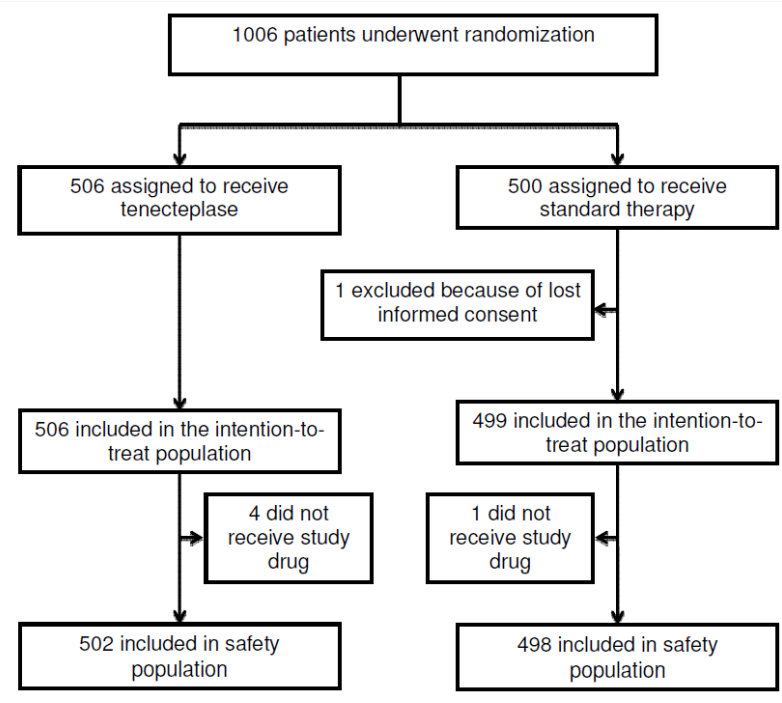

Figura 3. Randomização: 1006 pacientes foram acompanhados no período de 2007 a 2011, em 76 centros localizados em 13 países diferentes (Meyer, 2014).

Em relação à investigação etiológica dos eventos trombóticos, sabemos que o fenômeno da trombogênese repousa sobre três pilares, de acordo com a tríade clássica de Virchow: 1) estase sanguínea; 2) lesão endotelial; 3) estado de hipercoagulabilidade. No caso da paciente em questão, não havia história de imobilização prolongada ou condições sistêmicas que justificassem lesões endoteliais, ficando questionada uma possível trombofilia hereditária ou adquirida, a qual pudesse ser precipitada pelo uso de anticoncepcional.

Pacientes com TEP ou TVP sem fator óbvio desencadeante devem ser submetidos à dosagem de anticorpos antilúpico, anticardiolipina e Beta-2Glicoproteína - que, na paciente relatada, vieram negativos.

Já as trombofilias hereditárias são condições genéticas, determinadas por ganho ou perda de função de alguma proteína. Os ganhos de função se resumem às mutações de Fator $\mathrm{V}$ de Leiden e Protrombina G20210A, sendo essas responsáveis pela maior parte das trombofilias, cerca de 50 a $60 \%$ do total. As perdas de função afetam essencialmente a Antitrombina III, Proteína C e Proteína S, que perfazem $40 \%$ das trombofilias. Disfibrinogenemias também podem predispor a eventos trombóticos, mas são raras.

Em pacientes sem história pregressa de trombose, com TEP isolado como manifestação inicial, a incidência de trombofilias hereditárias é maior do que na população geral. Esses achados foram relatados por Pomero et al. em 2014, que registraram diferença significativamente estatística $(p<0,0001)$ tanto para a Protrombina quanto para o Fator $\mathrm{V}$, em relação à população geral. ${ }^{7}$
A Diretriz do grupo NICE (2013) recomenda pesquisar trombofilias em pacientes jovens, com história familiar ou pessoal de trombose, especialmente se for recorrente ou em vasos atípicos. ${ }^{3}$ Tal pesquisa deve levar em conta a interação com anticoagulantes, que ocorre na Antitrombina III, Proteína C e Proteína S, sendo frequentemente responsável por falsos positivos. A associação com anticoncepcionais, suspeitada no caso da paciente aqui descrita, pode aumentar a resistência à Proteína $\mathrm{C}$ e potencializar ação trombogênica das mutações de protrombina e Fator $\mathrm{V}$ de Leiden.

Desse modo, é válida, mas não obrigatória, a testagem dessas mutações em mulheres jovens, usuárias de anticoncepcional hormonal oral, com evento trombótico inexplicado. Tal investigação frequentemente esbarra em questões financeiras, devido ao alto custo desses exames. Por isso, recomenda-se que a investigação de trombofilias seja realizada não de rotina, mas criteriosamente, avaliando as particularidades de cada caso.

\section{REFERÊNCIAS BIBLIOGRÁFICAS}

1. Choi WH, Kwon SU, Jwa YJ et al. The pulmonary embolism severity in-dex in predicting the prognosis of patients with pulmonary embolism. Korean J Intern Med. 2009 Jun;24(2):1237.

2. Daniels LB, Parker JA, Patel SR, Grodstein F. Relation of duration of symptoms with response to thrombolytic therapy in pulmonary embo-lism. Am J Cardiol 1997;80:184-8.

3. Howard LS, Hughes RJ. NICE guideline: management of venous thromboembolic diseases and role of thrombophilia testing. Thorax. 2013 Apr;68(4):391-3.

4. Jaff MR, McMurtry MS, Archer SL et al. Management of massive and submassive pulmonary embolism, iliofemoral deep vein thrombosis, and chronic thromboembolic pulmonary hypertension: a scientific statement from the American Heart Association. Circulation. 2011 April 26;123(16):1788-830.

5. Konstantinides S. Acute pulmonar embolism. N Engl J Med 2008; 359:2804-13.

6. Meyer G, Vicaut E, Danays T et al. Fibrinolysis for patients with inter-mediate-risk pulmonary embolism. N Engl J Med. 2014 Apr 10;370(15):1402-11.

7. Pomero $F$, Ageno $W$, Serraino $C$ et al. The role of inherited thrombophil-ia in patients with isolated pulmonary embolism: a systematic review and a meta-analysis of the literature. Thromb Res. 2014 Jul;134(1):84-9.

8. Silverstein MD1, Heit JA, Mohr DN et al. Trends in the incidence of deep vein thrombosis and pulmonary embolism: a 25-year population-based study. Arch Intern Med. 1998 Mar 23;158(6):585-93.

9. Torbicki A, Perrier A, Konstantinides SV, et al. Guidelines on the diagno-sis and management of acute pulmonary embolism: the Task Force for the Diagnosis and Management of Acute Pulmonary Embolism of the European Society of Cardiology (ESC). Eur Heart J 2008;29:2276-315.

10. Spencer FA1, Emery C, Lessard D et al. The Worcester Venous Throm-boembolism Study A Population-Based Study of the Clinical Epidemi-ology of Venous Thromboembolism. J Gen Intern Med. Jul 2006; 21(7): 722-727. 